\title{
Overexpression of CBL interacting protein kinase 2 improves plant tolerance to salinity and mercury
}

\author{
W.H. PAN ${ }^{1}$, Z.Z. ZHENG ${ }^{2}$, X. YAN ${ }^{2,3}$, J.Q. $\mathrm{SHEN}^{2}$, J.X. SHOU ${ }^{1}$, L.X. JIANG ${ }^{4}$, and J.W. PAN ${ }^{3 *}$ \\ College of Life Sciences, Shaoxing University, Shaoxing, Zhejiang 312000, P.R. China ${ }^{1}$ \\ College of Chemistry and Life Sciences, Zhejiang Normal University, Jinhua, Zhejiang 321004, P.R. China ${ }^{2}$ \\ School of Life Sciences, Lanzhou University, Lanzhou 730000, P.R. China ${ }^{3}$ \\ College of Agriculture and Biotechnology, Zhejiang University, Hangzhou 310058, P.R. China
}

\begin{abstract}
In plants, calcineurin B-like proteins (CBLs) and CBL-interacting protein kinases (CIPKs) regulate $\mathrm{Ca}^{2+}$ signalling and so responses to biotic and abiotic stresses. However, the details of specific CIPKs functions in various stress responses are poorly understood. Here, we report roles of dicot and monocot CIPK2 genes in response to salinity and heavy metals. Arabidopsis thaliana AtCIPK2 was found to be universally expressed in different tissues and organs and furthermore induced by salinity. Overexpression of AtCIPK2 or Tibetan Plateau wild barley (Hordeum spontaneum) HsCIPK2 in Arabidopsis alleviated toxic effects of $\mathrm{NaCl}$ and mercury on seed germination and root growth. Similarly, reduced toxic effects of copper and cadmium on seed germination, but not on root growth, were observed in these transgenic lines. Live-cell fluorescence imaging analysis revealed that HsCIPK2 was predominantly distributed in the cytoplasm and nucleus and weakly localized at the plasma membrane (PM), but its PM association was rapidly enhanced upon exposure to high salinity and mercury. These results suggest an involvement of CIPK2 in plant tolerance to salinity and mercury and provide a new insight into physiological functions of CIPKs in plant response to heavy metals.
\end{abstract}

Additional key words: cadmium, copper, gene expression, $\mathrm{NaCl}$, root growth, seed germination, subcellular localization.

\section{Introduction}

To adapt to a changing environment, plants established a series of regulatory mechanisms, such as sensing, decoding, and transduction of various stress signals, stress-related gene expression, and final physiological responses. Calcium is a second messenger controlling a variety of actions including gene expression (Dodd et al. 2010). The change of calcium signals is initially sensed and decoded by calcium sensors and, in turn, calcium sensor interacting proteins transmit resulting signals to the downstream effectors to activate the expression of early response genes, finally leading to a wide range of physiological processes (Sanders et al. 2002, Batistič and

Kudla 2012, Zhu et al. 2013). In the past decade, calcineurin B-like proteins (CBLs), calcium sensor proteins, CBL-interacting protein kinases (CIPKs), and $\mathrm{Ca}^{2+}$-dependent serine/threonine kinases have been identified as a plant-specific signalling system that functions in biotic and abiotic stress responses (Kolukisaoglu et al. 2004, Xu et al. 2006, Luan 2009, Weinl and Kudla 2009, Hashimoto and Kudla 2011, De la Torre et al. 2013).

In Arabidopsis, overexpression of AtCIPK6 enhances plant tolerance to salt stress (Chen et al. 2013) while its down-regulation in mutants display a salt-hypersensitive

Submitted 10 January 2017, last revision 16 July 2018, accepted 1 August 2018.

Abbreviations: CaMV - cauliflower mosaic virus; CBLs - calcineurin B-like proteins; CDS - coding sequence; CIPKs CBL-interacting protein kinases; DAPI - 4',6-diamidino-2-phenylindole; FM4-64 - N-(3-triethylammoniumpropyl] -4[6-(4-(diethylamino)phenyl)hexatrienyl]pyridinium dibromide; GFP - green fluorescent protein; GUS - $\beta$-glucuronidase; MS - Murashige and Skoog; PM - plasma membrane; qPCR - quantitative polymerase chain reaction; RT-PCR - reverse transcription-PCR.

Acknowledgments: We thank Dr. G. Zhang (Zhejiang University, China) and Dr. Y. Yang (Pennsylvania State University, USA) for generously sharing published materials. This work was supported by the China National Key Programs for Transgenic Crops (No. 2009ZX08009-076B), the National Natural Science Foundation of China (Nos. 31670283 and 91754104), and the Natural Science Foundation of Zhejiang Province (Nos. LY17C060001, LY16C130004, and Y3100207). The first three authors contributed equally to this work.

* Corresponding author; e-mail: jwpan@1zu.edu.cn 
phenotype (Tripathi et al. 2009). In Oryza sativa, overexpression of $O s C I P K 3, O s C I P K 12$, and OsCIPK 15 improves the resistance to cold, drought, and salt stresses (Xiang et al. 2007), while loss-of-function OsCIPK31 mutants exhibit hypersensitive responses to multiple abiotic stresses including abscisic acid (ABA), salt, mannitol, and glucose during seed germination and early seedling stages (Piao et al. 2010). Overexpression of Zea mays ZmCIPK16 or Hordeum brevisubulatum HbCIPK2 in Arabidopsis sos 2 mutants improves their tolerance to salt and/or osmotic stresses (Zhao et al. 2009, Li et al. 2012). Furthermore, expression of halophytic shrub (Nitraria tangutorum) NtCIPK2 in Escherichia coli improves cell tolerance to high salinity, alkalinity, osmotic stress, dehydration, heat, and cold stresses (Zheng et al. 2014).

In addition to the roles of CIPKs in above-mentioned various stresses, however, it is no evidence showing that CIPKs function in plant response to heavy metal toxicity.

As a result of industrial activity, heavy metal contamination in soil and water has become a major environmental concern due to its impacts on plant growth and yield. Heavy metals are commonly categorized as essential and non-essential ones on the basis of their roles in plant growth (DalCorso et al. 2013, Sharma et al. 2016). Essential heavy metals (e.g. $\mathrm{Cu}^{2+}, \mathrm{Zn}^{2+}, \mathrm{Mn}^{2+}, \mathrm{Fe}^{2+}, \mathrm{Ni}^{2+}$, and $\mathrm{Co}^{2+}$ ) at low concentrations are necessary for plant growth, but their excessive amounts are toxic to plant cells whereas non-essential heavy metals $\left(\right.$ e.g. $\mathrm{Hg}^{2+}, \mathrm{Cd}^{2+}$, and $\mathrm{Pb}^{2+}$ ) are more hazardous to plant growth even at low concentrations. The aim of this work was to characterize the physiological role of Arabidopsis AtCIPK2 and wild barley HsCIPK2 in response to heavy metals and salinity.

\section{Materials and methods}

Plants and growth conditions: Arabidopsis thaliana L. (ecotype Columbia-0) seeds were surface sterilized and imbibed in the dark at $4{ }^{\circ} \mathrm{C}$ for $3 \mathrm{~d}$ and then sown onto $0.5 \times$ Murashige and Skoog (MS) agar plates $[1.5 \%(\mathrm{~m} / \mathrm{v})$ agar and $1 \%(\mathrm{~m} / \mathrm{v})$ sucrose, $\mathrm{pH}$ 5.6]. Seedlings, unless specified, were vertically grown on agar plates in a growth room $\left(22 / 20^{\circ} \mathrm{C}\right.$ day/night temperature, $16 / 8$-h photoperiod, $80 \mu \mathrm{mol} \mathrm{m} \mathrm{m}^{-2} \mathrm{~s}^{-1}$ irradiance, and $70 \%$ air humidity; for details see Wang et al. 2013). Grains of Tibetan Plateau annual wild barley (Hordeum spontaneum C. Koch) cv. X74, were surface sterilized and subsequently germinated between layers of wet filter papers with $0.1 \mathrm{mM} \mathrm{CaCl}_{2}$ (pH 5.8) in the dark and $25^{\circ} \mathrm{C}$ for $1 \mathrm{~d}$ and then germinated grains were incubated in $0.1 \mathrm{mM} \mathrm{CaCl}_{2}$ solution for $4 \mathrm{~d}$ (Pan et al. 2004).

Bioinformatic analysis, cloning, and constructs of HsCIPK2 and AtCIPK2: Full-length cDNAs of AtCIPK2 (AAF86506; At5G07070) and OsCIPK2 (ACD76974) as probes were used to search their homologous gene in full-length cDNA library of Hordeum vulgare (http://earth.lab.nig.ac.jp/ dclust/cgi-bin/barley_pub/).

Based on a cDNA sequence with the highest identity with AtCIPK2 and OsCIPK2, reverse transcription (RT)-PCR was used to amplify full-length coding sequence (CDS) of $H_{S} C I P K 2$. The total RNA of wild barley was isolated by RNeasy plant mini kit (Qiagen, Valencia, CA, USA) and subsequently, the first-strand of cDNA was synthesized with SuperScript III first-strand synthesis kit (Invitrogen, Carlsbad, CA, USA), as previously described (Wang et al. 2016). Bioinformatic analyses of CIPK 2 including amino acid alignment and transmembrane domain prediction were performed using Lasergene software (MegAlign) and TMHMM2.0 server (http://www.cbs.dtu.dk/services/ TMHMM-2.0/), respectively.

Constructs of AtCIPK2 and HsCIPK2 under the control of the constitutive cauliflower mosaic virus (CaMV) 35S promoter were generated using PCR

amplification with gene-specific primers (Table 1 Suppl.), restriction digestion, and ligation reaction with transformation vector (pCAMBIA2300) containing $2 \times$ $35 \mathrm{~S}$ promoter. Vectors pBI121 with GUS (encoding $\beta$-glucuronidase) and pCAMBIA1300 with $35 \mathrm{~S}$ promoter and GFP (encoding green fluorescent protein; Wang et al. 2013) were used to generate the ProAtCIPK2:GUS construct (1381 bp; Table 1 Suppl.) and the constructs of Pro35S:HsCIPK2-GFP and Pro35S:GFP (as a negative control; Table 1 Suppl.), respectively. All resulting constructs were confirmed by sequencing and consequently transformed into wild-type Arabidopsis ecotype (Col-0) via Agrobacterium-mediated floral dipping.

PCR-based gene expression assay: Reverse transcription (RT) PCR was used to examine whether exogenous genes are expressed in Arabidopsis transgenic lines. The total RNA isolation from T2 kanamycin-resistant transgenic lines and the first-strand cDNA synthesis were carried out as described above. The cDNA templates were used in PCR amplification for AtCIPK2 (34 cycles), HsCIPK2 (34 cycles), and AtActin11 (AT3G12110; 28 cycles; as an internal control) transcripts with the gene-specific primers (Table 1 Suppl.). PCR products were analyzed by $1 \%$ $(\mathrm{m} / \mathrm{v})$ agarose gel electrophoresis.

Real-time quantitative (q) PCR was performed with Thunderbird SYBR qPCR mix (Toyobo, Japan) and StepOnePlus real-time PCR system (Applied Biosystems, Foster City, CA, USA). Reactions were performed in a $20-\mathrm{mm}^{3}$ volume contained $10 \mathrm{~mm}^{3}$ of $2 \times$ SYBR qPCR mix (Toyobo), $40 \mathrm{ng} \mathrm{cDNA}$, and $1 \mu \mathrm{M}$ of gene-specific primer (Table 1 Suppl.). PCR cycles were performed as: $95^{\circ} \mathrm{C}$ for $5 \mathrm{~min} ; 40$ cycles of $95{ }^{\circ} \mathrm{C}$ for $5 \mathrm{~s}$ and $60^{\circ} \mathrm{C}$ for $45 \mathrm{~s}$. The resulting data were collected and analyzed using the StepOne software $v 2.1$ and consequently normalized with the housekeeping gene AtActin2 (At3G18780) (Table 1 Suppl.). The transcriptions in all treatments with $\mathrm{NaCl}$ or 
$\mathrm{HgCl}_{2}$ were standardized to the corresponding mock control or 0 min time point.

Determination of seed germination and seedling growth: Seeds of Col-0 and two independent homozygous transgenic lines overexpressing $H_{S} C I P K 2$ and AtCIPK2 (T3 generation) after 3 -d cold treatment were sown on $0.5 \times \mathrm{MS}$ agar plates $(0.7 \%$ agar and $1 \%$ sucrose, $\mathrm{m} / \mathrm{v}, \mathrm{pH}$ 5.6) supplemented with different concentrations of $\mathrm{NaCl}$, anhydrous $\mathrm{HgCl}_{2}, \mathrm{CuSO}_{4} \cdot 5 \mathrm{H}_{2} \mathrm{O}$, and anhydrous $\mathrm{CdCl}_{2}$. Agar plates were horizontally incubated for $7 \mathrm{~d}$ in the growth room. Seed germination rates [\%] were calculated by a formula: the number of germinated seeds/the total number of seeds $\times 100$. All germination rates were standardized to the corresponding mock control $(100 \%)$ and thereby presented as relative germination rates.

To assay the effects of $\mathrm{NaCl}$ on root growth, two methods were used: 5-d-old vertically grown seedlings were transferred to fresh $0.5 \times \mathrm{MS}$ agar plates supplemented with $125 \mathrm{mM} \mathrm{NaCl}$, and subsequently rotated for $180^{\circ}$ (roots vertically upward) and vertically incubated for additional two weeks before imaging; alternatively, 5-d-old vertically grown seedlings were transferred to the same agar plates supplemented with 125 $\mathrm{mM} \mathrm{NaCl}$, and subsequently vertically incubated for another $3 \mathrm{~d}$ without $180^{\circ}$ rotation before imaging, the latter of which was also similarly used to examine the effects of heavy metals on root growth. For quantification of root growth, root elongation of the primary roots was measured during 2-week or 3-d incubation using ImageJ software (http://rsb.info.nih.gov/ij). Average values of root elongation were standardized to the corresponding mock control and presented as relative root elongation (\%). In addition, a total fresh mass of seedlings in each treatment and an average fresh mass per seedling were presented.

Confocal microscopy and quantification: To analyze the subcellular localization of HsCIPK2-GFP, 5-d-old vertically grown seedlings of transgenic lines expressing $H S C I P K 2-G F P$ or free GFP were incubated in $0.5 \times \mathrm{MS}$ liquid medium containing $5 \mu \mathrm{g} \mathrm{cm} \mathrm{cm}^{-3}$ 4',6-diamidino2-phenylindole (DAPI) in darkness for $10 \mathrm{~min}$, washed with fresh $0.5 \times$ MS liquid medium, and then incubated for $2 \mathrm{~min}$ in $0.5 \times \mathrm{MS}$ liquid medium containing $1 \mu \mathrm{M}$ N-(3-triethylammoniumpropyl]-4[6-(4-(diethylamino)phe nyl)hexatrienyl] pyridinium dibromide (FM4-64; Wang

\section{Results}

Although previous studies have shown that wild barley HbCIPK2 is involved in salt and osmotic stress tolerance (Li et al. 2012), tissue-specific expression pattern of CIPK2 in plants remains unknown. To this end, we generated transgenic lines expressing GUS under control of AtCIPK2 native promoter (ProAtCIPK2:GUS) in Arabidopsis. As shown in Fig. 1, GUS signals were detectable in the root (root-shoot junction and root tip), hypocotyl, cotyledon, stem, rosette leaf, and flower organs et al. 2013) before confocal imaging.

To determine the effects of $\mathrm{NaCl}$ and $\mathrm{Hg}^{2+}$ on subcellular distribution of HsCIPK2-GFP, 5-d-old vertically grown seedlings expressing $H S C I P K 2-G F P$ or free $G F P$ were incubated in $0.5 \times \mathrm{MS}$ liquid medium supplemented with $200 \mathrm{mM} \mathrm{NaCl}$ or $1 \mu \mathrm{M} \mathrm{HgCl}_{2}$ for different duration (as indicated in the figure legends) before confocal imaging.

Images were captured using confocal laser scanning microscope (Leica TCS SP5 AOBS, Bensheim, Germany). Excitation wavelengths were $488 \mathrm{~nm}$ (argon laser) for GFP, $543 \mathrm{~nm}$ (helium/neon laser) for FM4-64, and $355 \mathrm{~nm}$ (UV laser) for DAPI. Emission was detected at 496 - 532 nm for GFP, 651 - $761 \mathrm{~nm}$ for FM4-64, and 420 - $470 \mathrm{~nm}$ for DAPI. For quantification of GFP fluorescence intensities, laser, pinhole and gain settings of the confocal microscope were identical in the same treatment or genotype.

To measure the intensities of GFP fluorescence signals at the plasma membrane (PM), digital images were analyzed by regions of interest (ROIs) using a freehand line tool of ImageJ software as shown in Fig. 6 and relative fluorescence intensities were presented as percentages of the corresponding 30-min mock control (100\%) as previously described (Wang et al. 2016).

GUS staining assay: After 3 -d cold treatment in darkness, seeds were sown on $0.5 \times$ MS $1.5 \%(\mathrm{~m} / \mathrm{v})$ agar plates supplemented with different concentrations of $\mathrm{NaCl}$ as indicated in the text. Seven-d-old whole seedlings treated with or without $\mathrm{NaCl}$ or tissues and organs at different developmental stages of plants grown in the soil were vacuumed for $15 \mathrm{~min}$ in X-gluc staining solution [10 $\mathrm{mM}$ EDTA, $0.1 \%(? / \mathrm{v})$ Triton $X-100,2 \mathrm{mM} \mathrm{Fe}^{2+} \mathrm{CN}, 2 \mathrm{mM}$ $\mathrm{Fe}^{3+} \mathrm{CN}, 100 \mu \mathrm{g} \mathrm{cm}^{-3}$ chloramphenicol, $1 \mathrm{mg} \mathrm{cm}^{-3} \mathrm{X}$-gluc in $50 \mathrm{mM}$ phosphate buffer (pH 7.0)] (Yu et al. 2016) and subsequently incubated at $37^{\circ} \mathrm{C}$ overnight. After staining, resulting materials were stored in $70 \%(\mathrm{v} / \mathrm{v})$ ethanol solution before microscope imaging.

Statistical analyses: All quantitative data from at least three independent repeats were statistically evaluated using a Student's $t$-test (two tails; type 2). Numbers of seeds, seedlings, and cells examined are indicated in the figure legends.

including sepal, stamen (filament), pistil (stigma), and petal (base), indicating that $A t C I P K 2$ is universally expressed in various plant tissues and organs.

Next, to address whether salt stress induces the expression pattern of endogenous AtCIPK2 in Arabidopsis, a GUS staining assay was performed in the transgenic seedlings treated with different concentrations of $\mathrm{NaCl}(0,40,60$, and $80 \mathrm{mM})$. Salt treatment elevated the GUS signals in the root, hypocotyl, and cotyledon, 
especially in their vascular tissues, relative to the mock control (Fig. 2A-H). Consistently, RT-qPCR analysis showed that salt treatment enhanced the transcriptional expression of endogenous $A t C I P K 2$ in the seedlings under the same conditions (Fig. 2I). These results together demonstrate that $A t C I P K 2$ expression pattern is regulated by salt stress.

To characterize a role of $H S C I P K 2$ in response to various abiotic stresses, we used full-length cDNAs of OsCIPK2 and AtCIPK2 as a probe to perform a BLAST search for their homologue in full-length cDNA library of Hordeum vulgare and finally isolated HsCIPK2 CDS from the total cDNA pool of wild barley seedlings by RT-PCRand sequencing-based approaches. Alignment of the predicted amino acid sequence revealed that, similar to
OsCIPK2 and AtCIPK2, HsCIPK2 contained two CIPK-specific highly conversed domains including a $\mathrm{N}$-terminal kinase domain with an activation loop and a C-terminal regulatory domain with a NAF motif (Fig. 1 Suppl.). Furthermore, HsCIPK2 shared $\sim 86$ and $\sim 64 \%$ amino acid identity with OsCIPK2 and AtCIPK2, respectively, indicating that different monocot CIPK2 homologues display a higher level of evolutionary conservation, relative to dicot CIPK2. Consistent with this, a potentially conserved transmembrane helix domain in HsCIPK2 and OsCIPK2 but not in AtCIPK2 was predicted (Fig. 2 Suppl.). Sequencing of PCR products amplified from wild barley, rice, and Arabidopsis genomic DNAs (data not shown) confirmed the previous finding that plant-specific $C I P K 2$ does not have any introns $(\mathrm{Li}$
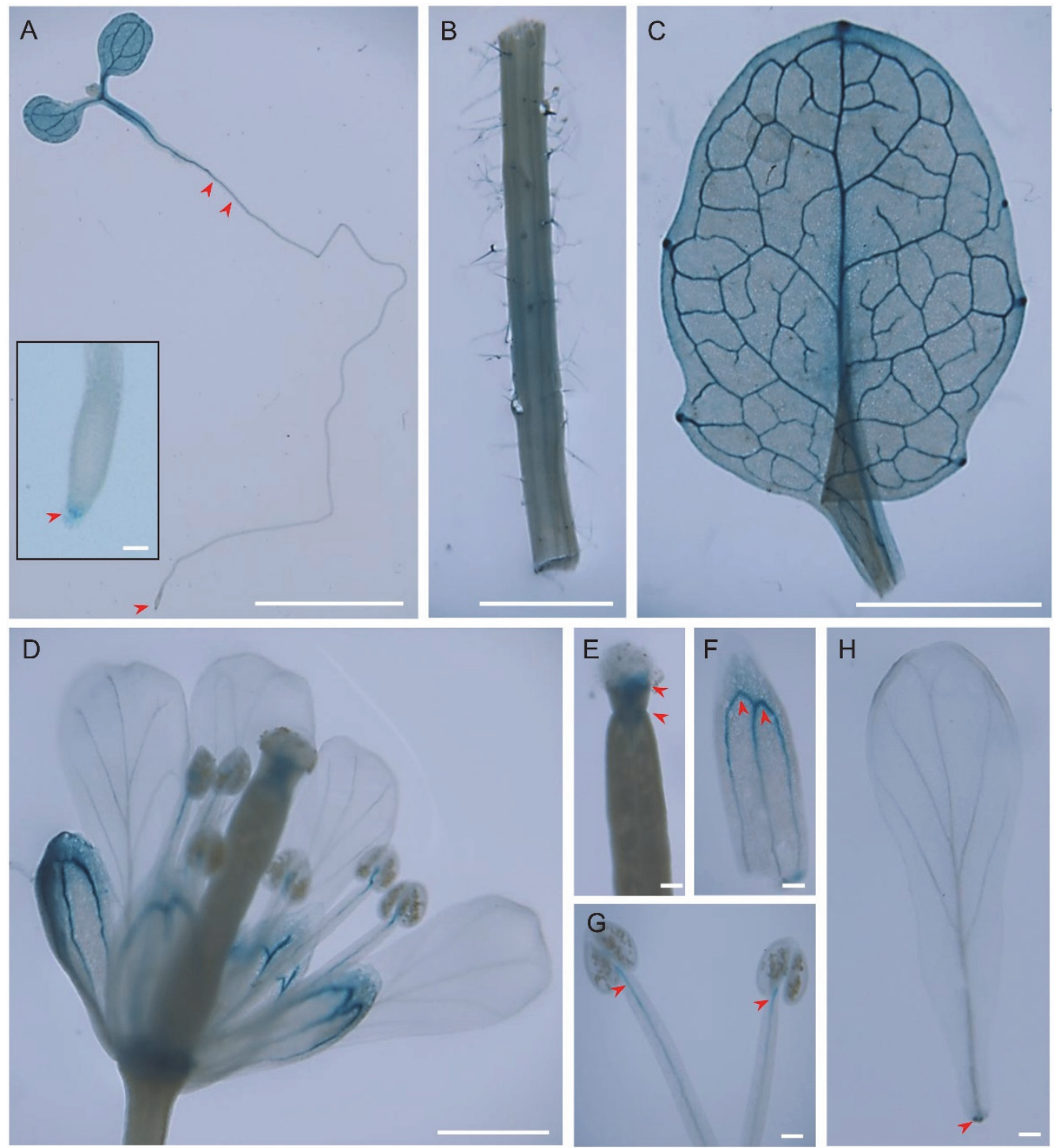

Fig. 1. GUS staining analysis of AtCIPK2 expression pattern. $A$ - Seven-d-old seedling expressing ProAtCIPK2: GUS. Inset shows GUS signals in the root tip. $B$ to $H$ - Individual organs including stem $(B)$, rosette leaf $(C)$, flower $(D)$, pistil and stigma $(E)$, sepal $(F)$, anthers and filaments $(G)$, and petal $(H)$ in soil-grown transgenic plants expressing ProAtCIPK2:GUS. Arrowheads show GUS signals in the root and flower organs. Bars $=5 \mathrm{~mm}(A), 200 \mu \mathrm{m}$ in inset $(A), 10 \mathrm{~mm}$ in $(B), 5 \mathrm{~mm}$ in $(C), 1 \mathrm{~mm}(D)$, and $200 \mu \mathrm{m}$ in $(E)$ to $(H)$. 
et al. 2012, Zheng et al. 2014). Thus, CDS and deduced amino acid sequence of $H S C I P K 2$, as a novel homologue of plant-specific CIPK2, were deposited into the GenBank (accession No. KP638475).

To address whether AtCIPK2 and HsCIPK2 are functionally involved in plant salt tolerance, transgenic lines overexpressing AtCIPK2 and HsCIPK2 under control of the 35S promoter (Pro35S:AtCIPK2; Pro35S:HsCIPK2; Fig. 3 Suppl.) were generated. Analysis of RT-PCR in the T2 generation showed that multiple transgenic lines displayed overexpression of AtCIPK2 or $H S C I P K 2$ relative to the wild-type control (Fig. 3 Suppl.). In T3 generation, two independent homozygous lines AtCIPK2-9, 2-18, and HsCIPK2-7, 2-15 were used to evaluate transgenic plant response to salt stress. In the absence of $\mathrm{NaCl}$, no significant differences were observed in seed germination and root growth between the transgenic lines and the wild type (Fig. $3 A, B$, Fig. 4A, $C$ Suppl., Fig. $5 A, B$ Suppl.). Upon seed exposure to 125 or $150 \mathrm{mM} \mathrm{NaCl}$, seed germination percentages in two independent transgenic lines overexpressing AtCIPK2 or $H s C I P K 2$ were higher than in the corresponding wild-type control (Fig. 3A, Fig. 5A Suppl.). Consistently, root growth in these two independent transgenic lines overexpressing AtCIPK 2 or $H s C I P K 2$ was more resistant to $125 \mathrm{mM} \mathrm{NaCl}$ relative to the corresponding wild-type grown on the vertical agar plates with $180^{\circ}$ rotation for additional two weeks (Fig. $3 B$; relative root elongation of Col-0: $11.8 \pm$ $5.5 \%$; AtCIPK2-9: $20.4 \pm 8.2 \%$; HsCIPK2-15: $24.0 \pm$ $7.3 \%$ ) or without $180^{\circ}$ rotation for another $3 \mathrm{~d}$ (Fig. $5 B$
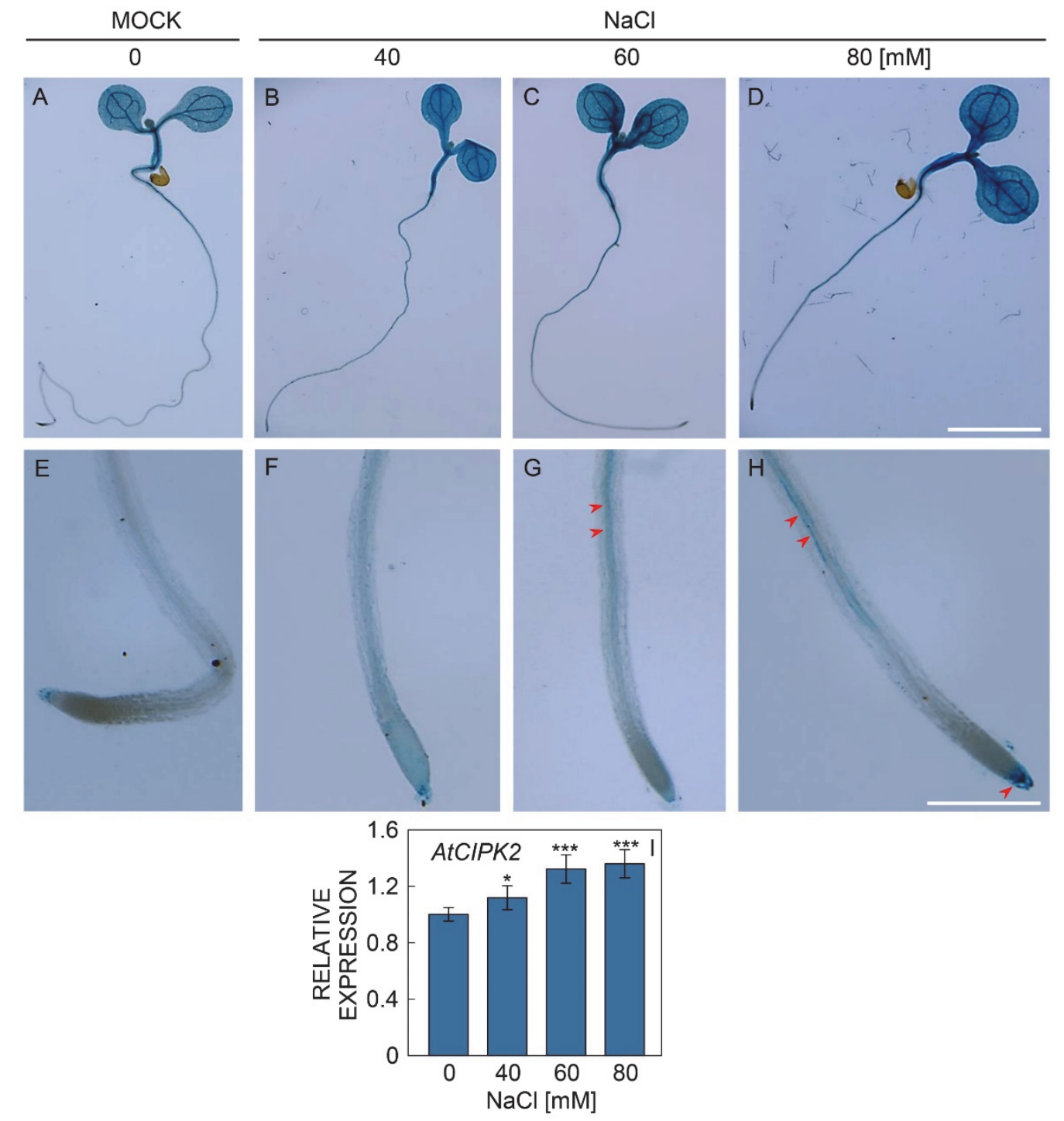

Fig. 2. Salt-induced expression pattern of $A t C I P K 2$. $A$ to $D$ - Whole seedlings. $E$ to $H$ - Magnified images of roots in $(A$ to $D)$. $I$ - real time qPCR. Seeds of ProAtCIPK2: GUS transgenic lines were germinated and vertically grown for $7 \mathrm{~d}$ on $0.5 \times$ MS agar plates supplemented with different concentrations of $\mathrm{NaCl}$ as indicated at the top panel before GUS staining. Arrowheads show salt-induced GUS signals in roots. Bars $=5 \mathrm{~mm}$ in $(A$ to $D)$ and $1 \mathrm{~mm}$ in $(E$ to $H)$. Means $\pm \mathrm{SD}, n=3$. Single and triple asterisks indicate significant differences at $P<0.01$ and 0.0001 , respectively (Student's $t$ test). 
W.H. PAN et al.
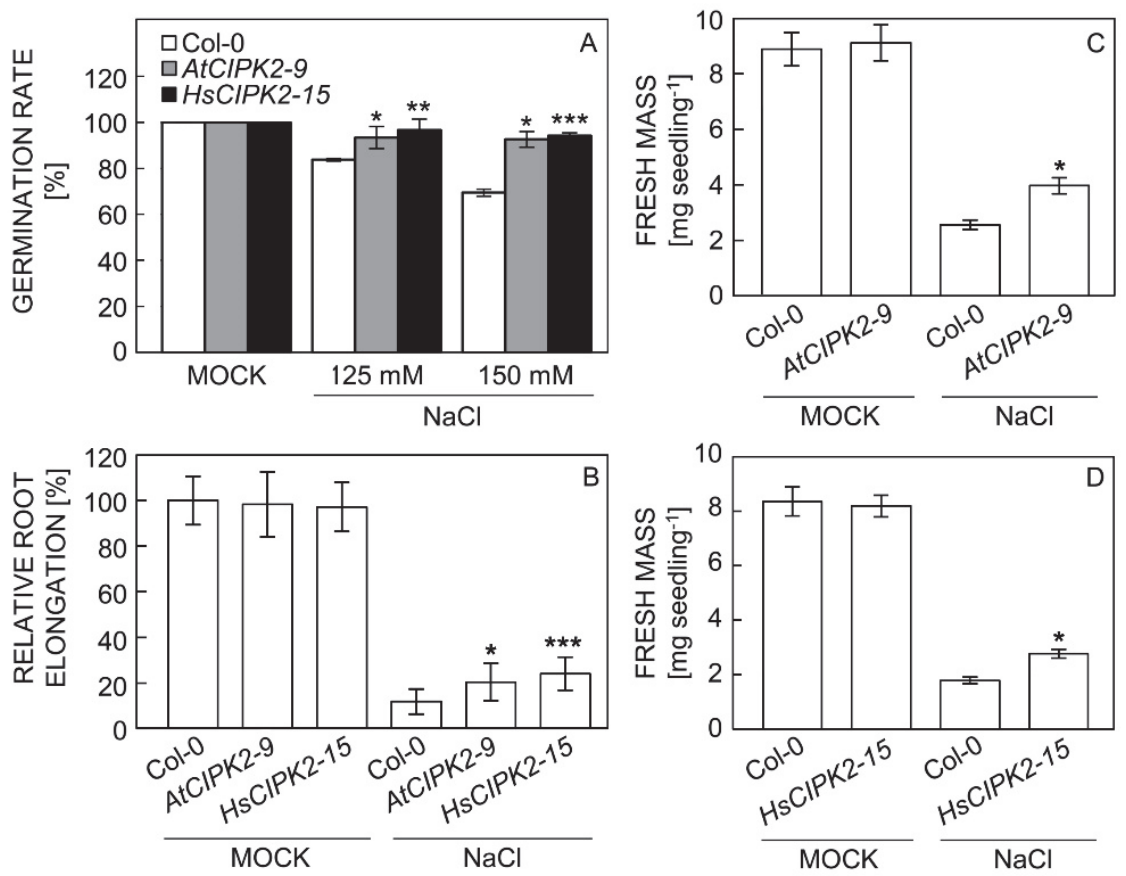

Fig. 3. Effects of salt stress on seed germination and seedling growth. $A$ - The wild-type, AtCIPK2-9, and HsCIPK2-15 seed germination rate after 7-d exposure to 125 or $150 \mathrm{mM} \mathrm{NaCl}$. $B$ to $D$ - Relative root eleongation and fresh mass of the wild-type, AtCIPK2-9, and HsCIPK2-15 seedlings after 2-week exposure to $125 \mathrm{mM} \mathrm{NaCl}$. Means \pm SDs, $n=120$ - 124 seeds or $24-30$ seedlings. Single, double, and triple asterisks indicate significant differences at $P<0.01,0.001$, and 0.0001 , respectively (Student's $t$ test; compared to the corresponding wild-type control).
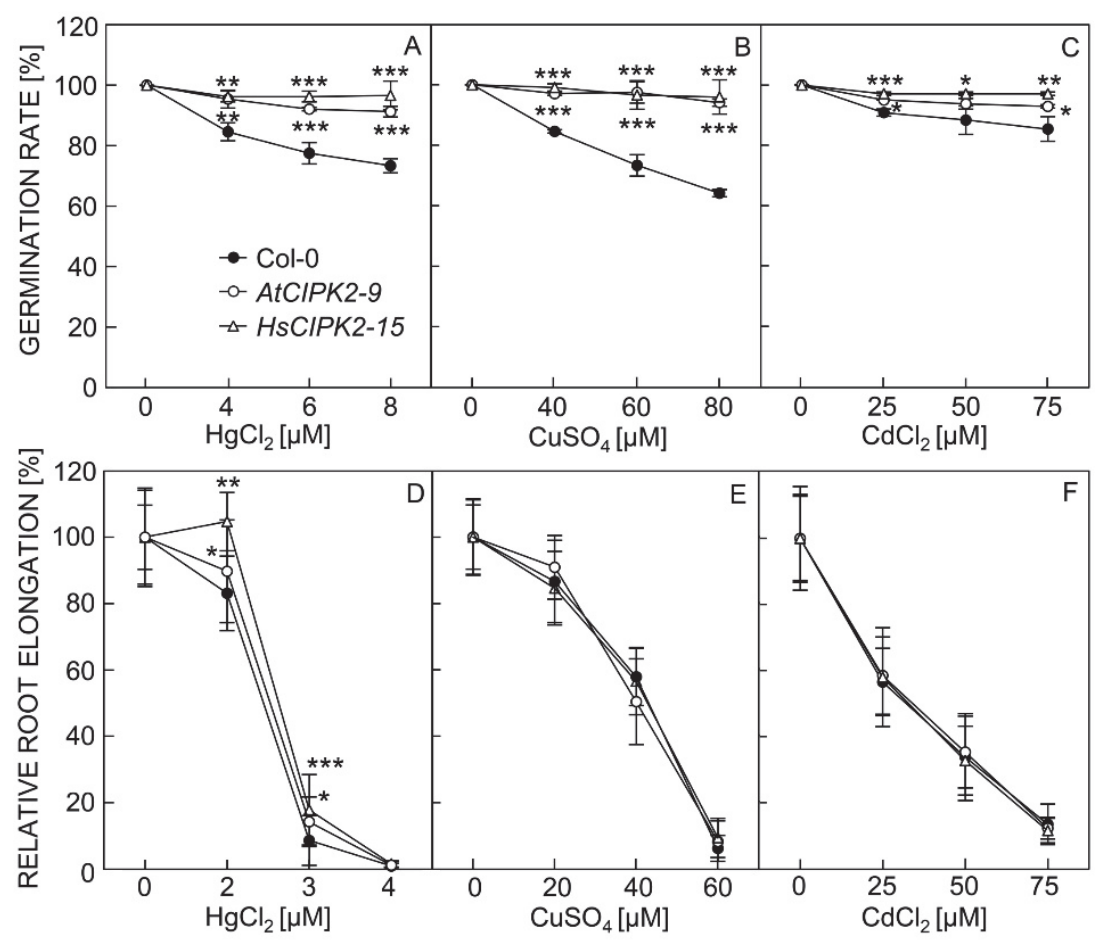

Fig. 4. Toxic effects of heavy metals on seed germination and root growth. $A$ to $C$-Seed germination assay in the wild-type, $A t C I P K 2-9$, and $H S C I P K 2-15$ seeds after 7-d exposure to heavy metal stresses. $D$ to $F$ - Root growth assay in the wild-type, AtCIPK2-9, and HsCIPK2-15 seedlings after 3-d exposure to heavy metal stresses. Relative germination rate (\%) of seeds $(n=100$ seeds each) and relative growth rate $(\%)$ of roots $(n=30-34$ seedlings each $)$ are presented. Means \pm SDs. Single, double, and triple asterisks indicate significan differences at $P<0.05,0.01$, and 0.001 , respectively (Student's $t$-test; compared to the corresponding wild-type control). 
Suppl.; Col-0: $21.8 \pm 3.7 \%$; AtCIPK2-18: $29.2 \pm 4.3 \%$; HSCIPK2-7: $34.2 \pm 4.0 \%$ ). Furthermore, the average fresh mass of transgenic plants was higher than that of the wild-type seedlings (Fig. 3C,D, Fig. 5C,D Suppl.). These results suggest that overexpression of AtCIPK2 and $H_{S C I P K 2}$ alleviates the inhibitory effect of $\mathrm{NaCl}$ on seed germination and seedling growth and thereby enhances salt tolerance.

Although plant-specific CIPKs are involved in multiple biotic and abiotic stresses (Shen et al. 2014), it remains unknown whether CIPKs function in plant responses to heavy metals. To test this, we examined the effects of heavy metals on seed germination and root growth in the transgenic lines overexpressing AtCIPK 2 or $H_{S C I P K 2}$. Upon seed exposure to $\mathrm{HgCl}_{2}(2,3,4,6$, and 8 $\mu \mathrm{M}), \mathrm{CuSO}_{4}(20,40,60$, and $80 \mu \mathrm{M})$, or $\mathrm{CdCl}_{2}(25,50$, and $75 \mu \mathrm{M})$, seed germination percentages in the wild type were significantly reduced (Fig. 4A-C, Fig. 6A-D Suppl.).
By contrast, the transgenic lines overexpressing AtCIPK2 or $H_{S} C I P K 2$ displayed higher seed germination percentages than the corresponding wild-type control (Fig. 4A-C, Fig 6A-L Suppl.). Consistently, root growth of the transgenic lines overexpressing AtCIPK2 or HsCIPK2 was more resistant to $2 \mu \mathrm{M} \mathrm{HgCl}_{2}$ (Fig. $4 D$; relative root elongation of Col-0: $83.1 \pm 11.2 \%$, AtCIPK2-9: $104.7 \pm$ $15.5 \%$; HsCIPK2-15: $89.1 \pm 8.8 \%$ ) and $3 \mu \mathrm{M} \mathrm{HgCl}_{2}$ (Fig. 4D; Col-0: $8.6 \pm 7.5 \%$, AtCIPK2-9: $17.9 \pm 7.5 \%$, $H s C I P K 2-15: 14.3 \pm 10.6 \%$ ) relative to the corresponding wild-type control. However, no significant differences were observed in root growth between these transgenic lines versus the wild-type seedlings in the presence of $\mathrm{CuSO}_{4}$ or $\mathrm{CdCl}_{2}$ (Fig. 4E,F). Together, these results suggest that overexpression of AtCIPK2 and HsCIPK2 improves seed germination tolerance to $\mathrm{Hg}^{2+}, \mathrm{Cu}^{2+}$, and $\mathrm{Cd}^{2+}$ and enhances root growth tolerance to $\mathrm{Hg}^{2+}$, but not to $\mathrm{Cu}^{2+}$ and $\mathrm{Cd}^{2+}$.
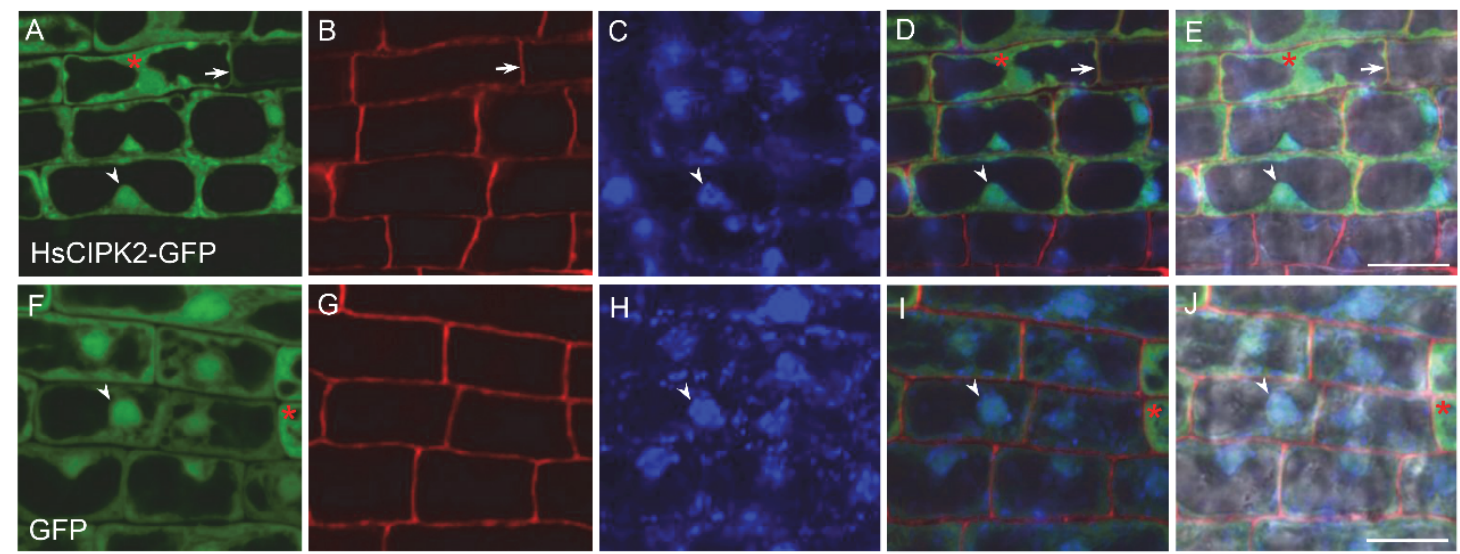

GFP

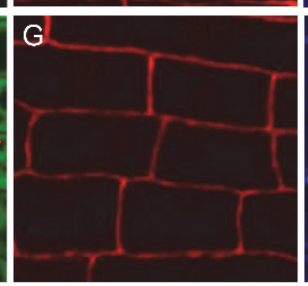

FM4-64

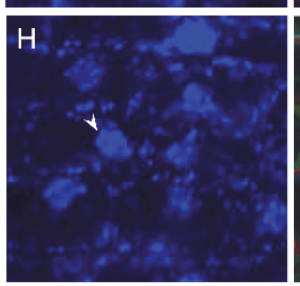

DAPI

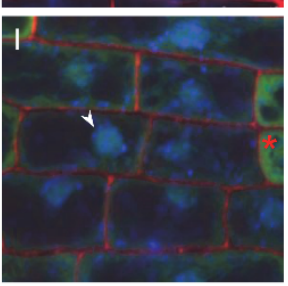

MERGED IN DARK FIELD

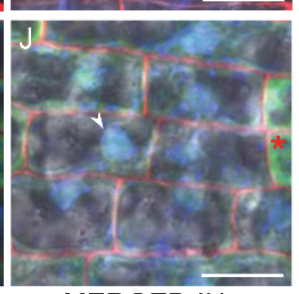

MERGED IN

LIGHT FIELD

Fig. 5. Live-cell imaging for subcellular distribution of HsCIPK2-GFP. $A$ to $E$-Subcellular localization of HsCIPK2-GFP in root epidermal cells. $F$ to $J$-Subcellular localization of free GFP in root epidermal cells. Arrowheads and arrows show co-localization of HsCIPK2-GFP or free GFP with DAPI-labeled nuclei and HsCIPK2-GFP with FM4-64-labeled PM, respectively. Asterisks show cytoplasmic localization of HsCIPK2-GFP or free GFP. Bars $=10 \mu \mathrm{m}$.

To analyze the subcellular distribution of CIPK2, we generated the transgenic lines expressing $H S C I P K 2-G F P$ under control of the $35 \mathrm{~S}$ promoter (Pro35S: HsCIPK2-GFP). FM4-64, a lipophilic styryl dye and DAPI, a fluorescent dye widely used for nuclear staining, were used to label the plasma membrane (PM) and nucleus, respectively. Different from the prediction (Fig. 2 Suppl.), live-cell imaging showed HsCIPK2-GFP fluorescence signals predominantly in the cytoplasm and nucleus while only weakly at the PMs in root epidermal cells (Fig. 5A-E). Similarly, the fluorescence signals of free GFP were observed in the cytoplasm and nucleus but not at PM (Fig. $5 F-J)$.

To address whether salt and mercury stresses influence subcellular localization of CIPK2 and/or its gene expression, we first used live-cell imaging to examine subcellular distribution of HsCIPK2-GFP upon seedling exposure to $200 \mathrm{mM} \mathrm{NaCl}$ or $1 \mu \mathrm{M} \mathrm{HgCl}_{2}$ for 10 - $90 \mathrm{~min}$.
Both treatments caused a rapid increase in HsCIPK2-GFP fluorescence signals in the PM, cytoplasm, and nucleus of root cells (Fig. 6A-E, $K-O$ ). By contrast, the fluorescence signals of free GFP were greatly reduced after exposure to high salinity or $\mathrm{HgCl}_{2}$ (Fig. $6 F-J, P-T$ ). Furthermore, quantitative analysis revealed that, upon 10-min exposure to $200 \mathrm{mM} \mathrm{NaCl}$ or $1 \mu \mathrm{M} \mathrm{HgCl}$, the PM-associated HsCIPK2-GFP fluorescence signals increased by approximately 20 and $50 \%$ relative to the mock controls, respectively, and subsequently reached a peak (increased by $\sim 50$ and $\sim 70 \%$, respectively) after 60 -min exposure (Fig. 6U,V).

Next, we performed a real-time qPCR analysis to examine whether salt- and mercury-induced PM association of HsCIPK2-GFP occurs via a transcriptional process. Although $\mathrm{NaCl}$ and $\mathrm{HgCl}_{2}$ treatments indeed enhanced the expression of endogenous AtCIPK2 (Fig. 2I, Fig. $7 A$ ), these treatments did not alter the expression of 
$35 \mathrm{~S}$ promoter-controlled $H S C I P K 2-G F P$ relative to the corresponding 0 -min time point (Fig. $7 B, C$ ). It was consistent with previous findings that the $35 \mathrm{~S}$ promoter does not respond to $\mathrm{NaCl}$ (Zhou et al. 2006, Sun et al.
2010) or $\mathrm{HgCl}_{2}$ (Shen et al. 2011). Taken together, these results suggest that $\mathrm{NaCl}$ or $\mathrm{HgCl}_{2}$ rapidly promote $\mathrm{PM}$ association of HsCIPK2.

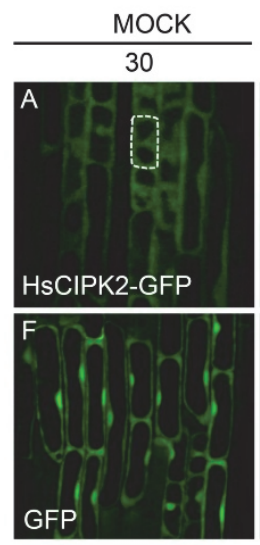

MOCK
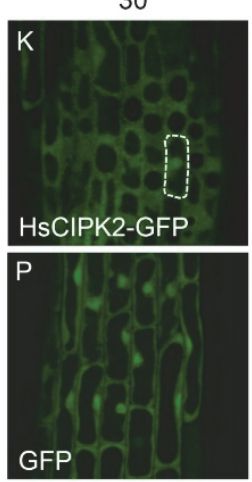
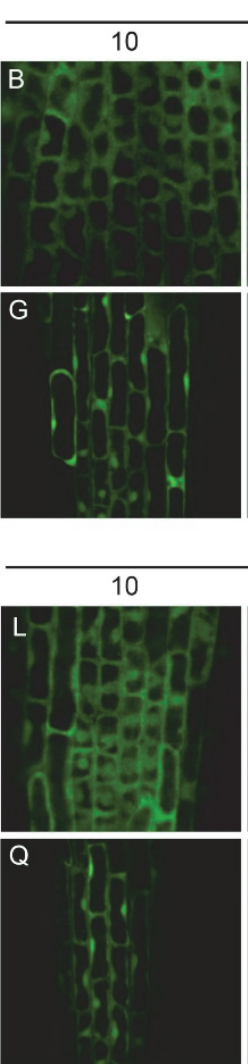

$200 \mathrm{mM} \mathrm{NaCl}$
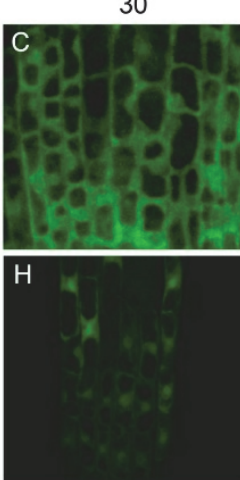

$1 \mu \mathrm{M} \mathrm{HgCl}_{2}$
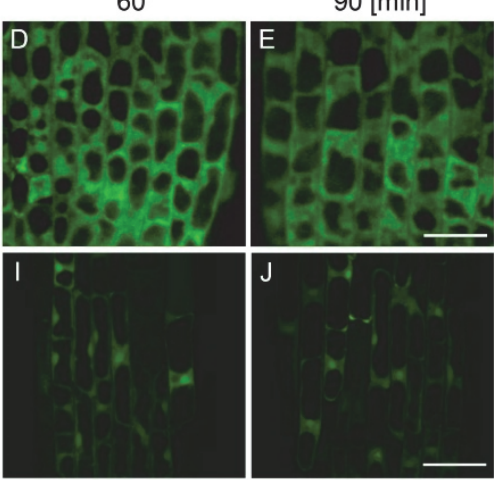

60
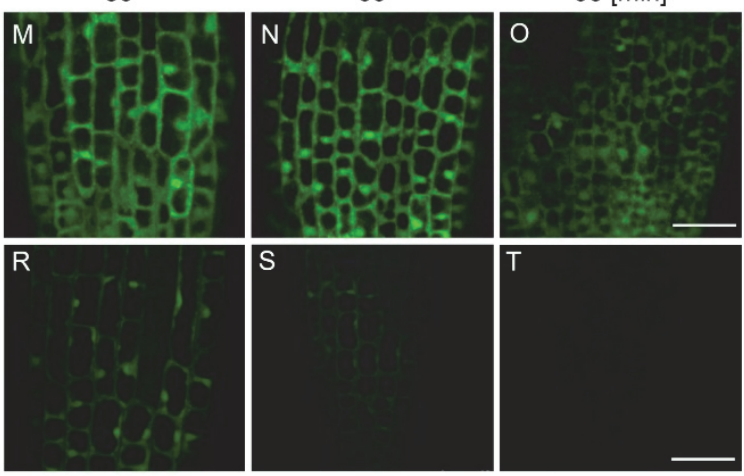

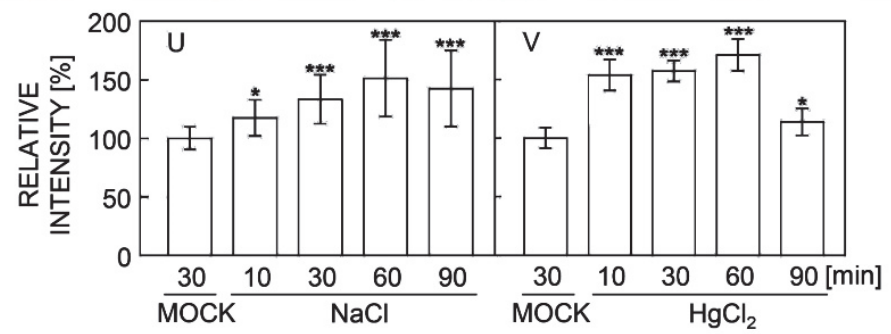

Fig. 6. Effects of $200 \mathrm{mM} \mathrm{NaCl}$ and $1 \mu \mathrm{M} \mathrm{HgCl}_{2}$ on subcellular distribution of HsCIPK2-GFP or GFP at different time points $(A$ to $T)$. Dashed boxes in $(A$ and $K$ ) show places for quantification of HsCIPK2-GFP signal intensities at the PM. Bars $=25 \mu \mathrm{m}$. $U$ and $V$ Quantification of PM-associated HsCIPK2-GFP signal intensities $(U ; n=150-185$ cells from 5 - 8 roots each; $V ; n=95-121$ cells from 5 roots each). Means \pm SDs. Single and triple asterisks indicate significant differences at $P<0.05$ and 0.001 , respectively (Student's $t$-test; compared to the mock control).

\section{Discussion}

Recently, increasing evidence has shown that multiple CIPKs are involved in plant response to biotic and abiotic stresses. Previous studies have shown that overexpression of wild barley HbCIPK2 in Arabidopsis sos2/cipk24 mutants ( $\mathrm{Li}$ et al. 2012) can rescue the salt-sensitive phenotype of Arabidopsis mutant sos 2-1, indicating that CIPK2 and CIPK24 may have an overlapping function in salt tolerance. Whether CIPK2 really possesses a significant role in the wild-type plant cell response to salt stress, however, remains unknown. Our findings showing that overexpression of AtCIPK2 and HsCIPK2 in Arabidopsis transgenic plants significantly enhances the tolerance of seed germination and plant growth to $\mathrm{NaCl}$ stress, together with the previous finding that expression of halophytic shrub NtCIPK2 in E. coli cells enhances host cell tolerance to salt toxicity (Zheng et al. 2014), 


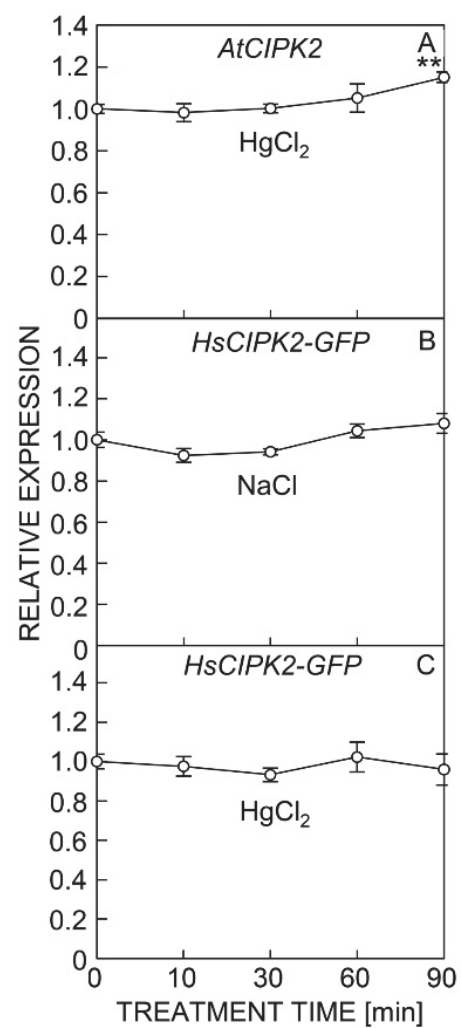

Fig. 7. RT-qPCR analysis of transcriptional levels of endogenous AtCIPK2 and exogenous HSCIPK2-GFP. A - The effect of mercury toxicity on the transcription of $A t C I P K 2$. B and $C$ - The effects of $\mathrm{NaCl}(B)$ and $\mathrm{HgCl}_{2}(C)$ on the transcription of $H s C I P K 2-G F P$ in transgenic seedlings harboring Pro35S:HsCIPK2-GFP. Five-d-old vertically grown seedlings were treated with $200 \mathrm{mM} \mathrm{NaCl}(B)$ or $1 \mu \mathrm{M} \mathrm{HgCl}_{2}(A$ and $C)$ in $0.5 \times$ MS liquid medium for different time lengths as indicated. Shown are means $\pm \mathrm{SD}$. Double asterisks indicate significant difference at $P<0.01$ (Student's $t$ test; compared to the 0 -min time point).

\section{References}

Batistič, O., Kudla, J.: Analysis of calcium signaling pathways in plants. -Biochim. biophys. Acta 1820: 1283-1293, 2012.

Chen, L., Wang, Q.Q., Zhou, L., Ren, F., Li, D.D., Li, X.B.: Arabidopsis CBL-interacting protein kinase (CIPK6) is involved in plant response to salt/osmotic stress and ABA. Mol. Biol. Rep. 40: 4759-4767, 2013.

DalCorso, G., Manara, A., Furini, A.: An overview of heavy metal challenge in plants: from roots to shoots. - Metallomics 5: 1117-1132, 2013.

De la Torre, F., Gutiérrez-Beltrán, E., Pareja-Jaime, Y., Chakravarthy, S., Martin, G.B., Del Pozo, O.: The tomato calcium sensor $\mathrm{Cbl10}$ and its interacting protein kinase Cipk6 define a signaling pathway in plant immunity. - Plant Cell 25: 2748-2764, 2013.

Dodd, A.N., Kudla, J., Sanders, D.: The language of calcium signaling. - Annu. Rev. Plant Biol. 61: 593-620, 2010.

Guo, Y., Qiu, Q.S., Quintero, F.J., Pardo, J.M., Ohta, M., Zhang, C.Q., Schumaker, K.S., Zhu, J.K.: Transgenic evaluation of activated mutant alleles of SOS2 reveals a critical demonstrate that CIPK2 plays a significant role in plant salt tolerance. In addition, we also noticed that, based on seedling growth phenotype, the wild-type seedlings appear to be more sensitive to $\mathrm{NaCl}$ on $0.5 \times \mathrm{MS}$ agar plates $(1 \%$ sucrose) in our study than on $1 \times$ MS agar plates $(2 \%$ sucrose) in the previous studies (Guo et al. 2004, Li et al. 2012).

In addition to the role of CIPK2 in plant tolerance to salt stress, CIPK2 functions in plant responses to heavy metals. Our findings showed that overexpression of AtCIPK2 and HsCIPK2 in Arabidopsis improved the tolerance of seed germination to $\mathrm{Hg}^{2+}, \mathrm{Cu}^{2+}$, and $\mathrm{Cd}^{2+}$. These transgenic lines, however, displayed an enhanced tolerance of plant growth only to $\mathrm{Hg}^{2+}$ but not to $\mathrm{Cu}^{2+}$ and $\mathrm{Cd}^{2+}$. Likely, CIPK2 played a specific role in response of seedling growth to $\mathrm{Hg}^{2+}$, but we cannot rule out the possibility that CIPK2 may function in responses to other heavy metals unexamined in this study.

Live-cell imaging revealed that $200 \mathrm{mM} \mathrm{NaCl}$ and $1 \mu \mathrm{M} \mathrm{HgCl}{ }_{2}$ rapidly enhanced the PM association of HsCIPK2-GFP, indicating this as an important step for CIPK2 action mode during its mediation of stress signalling in the plant cell. Furthermore, our analyses of GUS staining and real-time qPCR showed that salt and mercury toxicities can activate the expression of $C I P K 2$, but not that of $35 \mathrm{~S}$ promoter-controlled HsCIPK2-GFP. These findings suggest that salt and mercury stresses may stimulate physiological functions of CIPK2 at both transcriptional and posttranscriptional levels.

Whether CIPK2 is involved in either heavy metal uptake or vacuolar detoxification or both remains to be experimentally elucidated. Further isolation and functional identification of CIPKs in different plant species and clarification of CIPK2 involvement in some biochemical and/or cellular processes of heavy metal toxicities will provide a better understanding of physiological roles of CIPKs during plant stress responses.

requirement for its kinase activity and C-terminal regulatory domain for salt tolerance in Arabidopsis thaliana. - Plant Cell 16: 435-449, 2004.

Hashimoto, K., Kudla, J.: Calcium decoding mechanisms in plants. - Biochimie 93: 2054-2059, 2011.

Kolukisaoglu, U., Weinl, S., Blazevic, D., Batistic, O., Kudla, J.: Calcium sensors and their interacting protein kinases: genomics of the Arabidopsis and rice CBL-CIPK signaling networks. - Plant Physiol. 134: 43-58, 2004.

Li, R., Zhang, J., Wu, G., Wang, H., Chen, Y., Wei, J.: HbCIPK2, a novel CBL-interacting protein kinase from halophyte Hordeum brevisubulatum, confers salt and osmotic stress tolerance. - Plant Cell Environ. 35: 1582-600, 2012.

Luan, S.: The CBL-CIPK network in plant calcium signaling. Trends Plant Sci. 14: 37-42, 2009.

Pan, J.W., Zheng, K., Ye, D., Yi, H.L., Jiang, Z.M., Jing, C.T., Pan, W.H., Zhu, M.Y.: Ultraweak luminescence and sister-chromatid exchanges in root tip cells serve as sensitive indicators for $\mathrm{Al}$ toxicity and tolerance in barley. - Plant Sci. 
167: 1391-1399, 2004

Piao, H.L., Xuan, Y.L., Park, S.H., Je, B.I., Park, S.J., Park, S.H., Kim, C.M., Huang, J., Wang, G.K., Kim, M.J.: OsCIPK31, a CBL-interacting protein kinase is involved in germination and seedling growth under abiotic stress conditions in rice plants. - Mol. Cell 30: 19-27, 2010.

Sanders, D., Pelloux, J., Brownlee, C., Harper, J.F.: Calcium at the crossroads of signaling. - Plant Cell 14 (Suppl): S401-S417, 2002.

Sharma, S.S., Dietz, K.J., Mimura, T.: Vacuolar compartmentalization as indispensable component of heavy metal detoxification in plants. - Plant Cell Environ. 39: 1112-1126, 2016.

Shen, J.Q., Zheng, Z.Z., Pan, W.H., Pan, J.W.: [Functions and action mechanisms of CBL-CIPK signaling system in plants.] - Plant Physiol. J. 50: 641-650, 2014. [In Chin., ab: E]

Shen, Q., Jiang, M., Li, H., Che, L.L., Yang, Z.M.: Expression of a Brassica napus heme oxygenase confers plant tolerance to mercury toxicity. - Plant Cell Environ. 34: 752-763, 2011

Sun, Q., Gao, F., Zhao, L., Li, K., Zhang, J.: Identification of a new 130 bp cis-acting element in the TsVP1 promoter involved in the salt stress response from Thellungiella halophila. - BMC Plant Biol. 10: 90, 2010.

Tripathi, V., Parasuraman, B., Laxmi, A., Chattopadhyay, D.: CIPK6, a CBL-interacting protein kinase is required for development and salt tolerance in plants. - Plant J. 58: 778-790, 2009.

Wang, C., Hu, T., Yan, X., Meng, T., Wang, Y., Wang, Q., Zhang, X., Gu, Y., Sánchez-Rodríguez, C., Gadeyne, A., Lin, J., Persson, S., Van Damme, D., Li, C., Bednarek, S.Y., Pan, J.: Differential regulation of clathrin and its adaptor proteins, AP-2 and the TPLATE complex, during their membrane recruitment in Arabidopsis. - Plant Physiol. 171: 215-229, 2016.
Wang, C., Yan, X., Chen, Q., Jiang, N., Fu, W., Ma, B.J., Liu, J.Z, Li, C.Y., Bednarek, S.Y., Pan, J.: Clathrin light chains regulate clathrin-mediated trafficking, auxin signaling, and development in Arabidopsis. - Plant Cell 25: 499-516, 2013.

Weinl, S., Kudla, J.: The CBL-CIPK $\mathrm{Ca}^{2+}$-decoding signaling network: function and perspectives. - New Phytol. 184: 517-528, 2009.

Xiang, Y., Huang, Y., Xiong, L.: Characterization of stress-responsive CIPK genes in rice for stress tolerance improvement. - Plant Physiol. 144: 1416-1428, 2007.

Xu, J., Li, H.D., Chen, L.Q., Wang, Y., Liu, L.L., He, L., Wu, W.H.: A protein kinase, interacting with two calcineurin B-like proteins, regulates $\mathrm{K}^{+}$transporter AKT1 in Arabidopsis. - Cell 125: 1347-1360, 2006.

Yu, Q., Zhang, Y., Wang, J., Yan, X., Wang, C., Xu, J., Pan, J.: Clathrin-mediated auxin efflux and maxima regulates hypocotyl hook formation and light-stimulated hook opening in Arabidopsis. - Mol. Plant 9: 101-112, 2016.

Zhao, J., Sun, Z., Zheng, J., Guo, X., Dong, Z., Huai, J., Gou, M., He, J., Jin, Y., Wang, J., Wang, G.: Cloning and characterization of a novel CBL-interacting protein kinase from maize. - Plant mol. Biol. 69: 661-674, 2009.

Zheng, L.L., Gao, Z., Wang, J., Zhang, H.R., Wang, Y.C.: Molecular cloning and functional characterization of a novel CBL-interacting protein kinase NtCIPK2 in the halophyte Nitraria tangutorum. - Genet. mol. Res. 13: 4716-4728, 2014.

Zhou, H.L., Cao, W.H., Cao, Y.R., Liu, J., Hao, Y.J., Zhang, J.S., Chen, S.Y.: Roles of ethylene receptor NTHK1 domains in plant growth, stress response and protein phosphorylation. -FEBS Lett. 580: 1239-1250, 2006.

Zhu, S., Zhou, X., Wu, X., Jiang, Z.: Structure and function of the CBL-CIPK $\mathrm{Ca}^{2+}$-decoding system in plant calcium signaling. - Plant mol. Biol. Rep. 31: 1193-1202, 2013. 